\title{
Learning to Select State Machines using Expert Advice on an Autonomous Robot
}

\author{
Brenna Argall \\ The Robotics Institute \\ Carnegie Mellon University \\ Pittsburgh, PA 15213 \\ bargall@cs.cmu.edu
}

\author{
Brett Browning \\ The Robotics Institute \\ Carnegie Mellon University \\ Pittsburgh, PA 15213 \\ brettbecs. cmu .edu
}

\author{
Manuela Veloso \\ Computer Science Department \\ Carnegie Mellon University \\ Pittsburgh, PA 15213 \\ mmv@cs. cmu . edu
}

\begin{abstract}
Hierarchical state machines have proven to be a powerful tool for controlling autonomous robots due to their flexibility and modularity. For most real robot implementations, however, it is often the case that the control hierarchy is hand-coded. As a result, the development process is often time intensive and error prone. In this paper, we explore the use of an experts learning approach, based on Auer and colleagues' Exp3 [1], to help overcome some of these limitations. In particular, we develop a modified learning algorithm, which we call $r E x p 3$, that exploits the structure provided by a control hierarchary by treating each state machine as an 'expert'. Our experiments validate the performance of $r \operatorname{Exp} 3$ on a real robot performing a task, and demonstrate that $r \operatorname{Exp} 3$ is able to quickly learn to select the best state machine expert to execute. Through our investigations in these environments, we identify a need for faster learning recovery when the relative performances of experts reorder, such as in response to a discrete environment change. We introduce a modified learning rule to improve the recovery rate in these situations and demonstrate through simulation experiments that rExp3 performs as well or better than Exp3 under such conditions.
\end{abstract}

\section{INTRODUCTION}

There are a number of established approaches for developing a control architecture for generating autonomous mobile robot behavior. A common and powerful approach is to use hierarchies of finite state machines [2], [5], [6], whereby control consists of finite state machines that operate in parallel, or that use other state machines as macros to generate complex actions. Although many frameworks exist, they typically require extensive hand-coding when applied to real robot control problems. That is, the designer is responsible for creating the state machine hierarchy, and the control policies and parameters encoded in each control state. This is a time consuming and error prone process and one that does not scale well with increasing complexity of the robot or tasks.

We seek practical learning techniques that can aid, or replace, this process, thereby enabling more complex robots and tasks. In this paper, we make two key contributions. First, we present an experts-based algorithm for learning to select between state machines that can be integrated with a working robot control hierarchy, built upon the algorithm Exp3 [1]. Our second contribution extends Exp3 further, which we here introduce as $r \operatorname{Exp} 3$, to enable a more rapid response to changes in expert performance that are of a discrete nature. To evaluate our approach, we explore the performance of the algorithm on a real robot platform, and compare its performance to that of Exp3 in simulation. The real robot implmenentation uses the Segway RMP robots [10] performing a task integral to robot soccer and makes use of a hierarchy of state machines that we call skills [6].

Our paper is structured in the following way. In the ensuing section, we describe hierarchical state machines, as applied to robot control, that will form the basis for this paper. We then describe the Exp3 algorithm, and its extension to integrate within a robot control architecture. We additionally outline our modified algorithm, called $r \operatorname{Exp} 3$, for enhanced responsiveness to discrete environment changes. Based on this, we present our implementation of $r \operatorname{Exp} 3$ on a real robot platform, and compare its performance to that of Exp3 in simulation. We then close with our conclusions.

\section{STATE MACHINES FOR ROBOT CONTROL}

In this section, we describe our use of hierarchical state machines for autonomous robot control. Alternate implementations of state-based control are described in [12]. We formally define a state machine for control as consisting of a set of control states $c s_{i} \in C S$. Each control state encodes a control policy $\pi_{i}$, which is a function of the robot's internal state and its beliefs about the world (i.e. is a result of its perception system). This policy $\pi_{i}$ determines which action $a_{i} \in A$ to take, when in control state $c s_{i}$.

Transitions between different control states occur as a function of the robot's beliefs. Additionally, this state machine may terminate (i.e. enter an absorbing state) with success or failure. A state machine may therefore be viewed as providing deliberative goal-driven behavior, that will either fail or succeed and achieve the goal. For ease of reference, and following [6], we will refer to such a state machine as a skill. Thus, the term skill in this paper is equivalent to a goal-directed state machine for control, and the two terms will be used interchangeably.

State machines may be arranged into hierarchies. Two actions types are available for selection by a control policy: complex or primitive. A complex action consists of calling another control policy, and thus passing control to another state machine. This builds a hierarchy of state machines (equivalently a hierarchy of skills). By contrast, primitive actions command the robot using its available control primitives (e.g. velocity control). Note, that state machines may 
also execute in parallel, with or without synchronization coupling their execution. However, this is beyond the scope of this paper and will not be considered further.

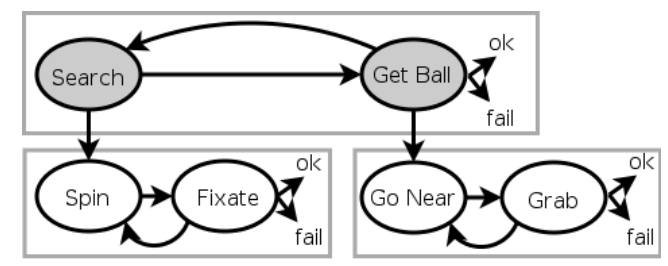

Fig. 1. An example soccer-related skill hierarchy. The robot will search for the ball, and then run up and grab it. Transitions are conditioned on the robot's perceptual beliefs.

State machines may transition to, or call, other state machines, thus building a skill hierarchy. Figure 1 shows a schematic of a small hierarchical state machine for controlling a robot performing a basic soccer skill. The robot's task is to grab the nearest ball it can find. It must first search for the ball, and run up to grab it once found. The power in a skill hierarchy lies in its task decomposition; that is, by enabling a larger task to be easily decomposed into subtasks that can be solved as independent problems (namely, search and get ball within this example). Moreover, each of the resulting skills may be reused for other similar problems. It is this 'divide and conquer' ability that makes state machines so useful for robot control. The drawback to this approach is that for a real robot, the control architecture is often hand coded. Typically, it is not task decomposition which is difficult. Rather, most of designer effort focuses on developing the control policies which call primitive actions (for example, the state go near in Fig. 1 calls a primitive velocity action before transitioning to grab). Moreover, the performance of skills using such policies is highly dependent on robot hardware and the environment.

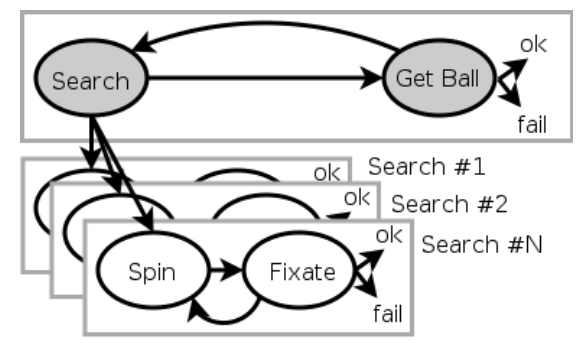

Fig. 2. Experts learning applied to state machines enables multiple skills to be developed, and the best one learned for a given environment.

In this paper, we aim to provide adaptability to the robot through the use of an experts learning algorithm. The key idea is as follows. Let us first view each state machine, or skill, as an expert that 'recommends' a control policy to achieve a particular goal. Suppose also, that we have multiple skills available for achieving the same goal. If these skills vary in their use of perception or more generally in their control policies, their performance characteristics (ie. success/failure rates) will be different. Thus, we can use expert learning to determine which 'expert' skill should be executed to maximize performance for the current domian. In summary, we can free up the designer to create a range of skills that have varying strengths and weaknesses, and let the expert learning automatically determine which skill should be run to achieve maximum performance (see Fig. 2).

\section{LEARNING WITH EXPERT ADVICE}

Expert learning (or the $k$-armed bandits problem, originally proposed by [11]) addresses the issue of choosing between multiple action recommenders, or experts, at each time step. Based upon the idea of slot machine gambling, the agent must choose an arm (expert) to pull, and then receives an associated pay off (reward). High reward, and therefore good performance, increases an expert's probability of being selected at each round.

We define formally a learning scenario where at each decision cycle, $k$, each of the $n$ experts makes a recommendation. The algorithm selects a single expert and executes the corresponding action, resulting in a payoff of $r^{k} \in \Re$. After $d$ decision cycles, a sequence of $r^{1}, r^{2}, \cdots, r^{d}$ payoffs have been rewarded. The aim of expert learning is to select the best expert over all decision cycles. We formulate this learning objective in terms of regret, where the regret at decision cycle $k$ is the difference between the observed reward $r^{k}$ of the selected action, and the reward $r_{b}^{k}$ we would have received from the best expert. Summed over all decision cycles,

$$
\text { Regret }=\sum_{k=1}^{d} r_{b}^{k}-\sum_{k=1}^{d} r^{k} \text {. }
$$

The goal of expert learning is to minimize this total regret.

When the chosen action executes on a real robot, however, only the reward for the recommending expert may be observed. Fortunately, the algorithm Exp3 ("Exponentialweight algorithm for Exploration and Exploitation”), introduced by Auer and colleagues in [1], addresses exactly this issue. Exp3 handles partial information games, where at each trial only the reward gained by the chosen expert, and not by every expert, is observed. The algorithm is a modification of the HEDGE experts learning algorithm [1], [8], which is founded upon the weighted majority algorithm of [9] and aggregating strategies of [13].

In Exp3, the reward earned at decision cycle $k$ is scaled inversely with the expert's selection probability $\operatorname{Pr}\left(x^{k}=\right.$ $i$ ) for that decision cycle, to compensate the fact that in a partial information game, experts with low probability are infrequently chosen and therefore have fewer observed rewards. Thus the reward earned at trial $k$ by expert $i$ is

$$
\hat{r}_{i}^{k}=\frac{r_{i}^{k}}{\operatorname{Pr}\left(x^{k}=i\right)}
$$

for the selected expert, and zero for all other experts. [1] proved that this regret approaches zero in the limit, assuming opponents with static policies.

More concretely, the probability of choosing expert $i$ at time step $k$ is governed by the normalizing equation

$$
\operatorname{Pr}\left(x^{k}=i\right)=\frac{w_{i}^{k-1}}{\sum_{j} w_{j}^{k-1}} .
$$

where $w_{i}^{k-1} \equiv e^{\sum_{k} \hat{r}_{i}^{k-1}}$ is the weight of expert $i$ at time 
$k-1$. Weights are initialized to be equal across experts, and the weight of an executed expert $i$ is updated according to:

$$
w_{i}^{k}=w_{i}^{k-1}\left(m_{i}^{k}\right)^{\frac{1}{\operatorname{Pr}\left(x^{k}=i\right)}} .
$$

Here the multiplier $m_{i}^{k} \equiv e^{r_{i}^{k}}$ follows the notation of [3] for update simplicity. Note that the product of the weight at decision cycle $k-1$ and the exponentiated multiplier at cycle $k$ is the same as adding $\hat{r}_{i}^{k}$ to $\sum_{k} \hat{r}_{i}^{k-1}$, and thus represents the cumulative reward received by expert $i$ up to trial $k$.

\section{EXPERTS LEARNING FOR SKILL SELECTION}

We now examine how to utilize expert learning in a hierarchical state machine (or skill hierarchy) for robot control. Concretely, we address the problem of learning to select the best skill, which we call skill selection. That is, from within our current execution of a skill, and given its current control state, which is the best state machine to call next (see Fig. 2). The idea behind this approach is that if a designer creates multiple skills to accomplish the same task, the robot will then be able to automatically determine the best skill to use given the current conditions.

We define the higher-level skill as the parent skill, and the state machines which it may call as the child skills. At each decision cycle, the parent skill chooses a new child skill to execute. Upon termination of the child skill, a reward is assigned to that skill. Its execution is evaluated based on success or failure; that is, the two absorbing states are assigned a payoff. Futhermore, each binary reward may be modified to incorporate a measure of execution quality. In our particular implementation, a quality measure based on execution time was added; faster executions earned more reward than slower executions. This modification was applied to the binary success reward exclusively, since the failure absorbing state was only entered after a timeout and thus all failed executions performed equally by this measure.

We thus have a framework ameniable to the experts learning approach; namely, each child skill is an expert that we select with some probability. In particular, we exploit the hierarchical state machine structure by tagging termination conditions with a reward. It is the existence of this structure which enables our learning. Based on the payoff from the selected expert/skill, we modify its future selection probability using equation (4). We validate this approach on a real robot in Section 5.

\section{A. Adapting to Discrete Performance Changes}

The algorithm Exp3 has an additional feature, that it promotes adaptability within non-stationary environments. In particular, we consider situations that cause a discrete, non-continuous change in the probability distribution which describes expert performance. That is, some change in the world causes the performance of each expert to change such that it reorders their relative selection probability ranking. For example, suppose expert A recommends command velocities for the robot which are best suited for smooth ground, while the recommendations of expert B are best suited for rough terrain. When operating indoors on a flat surface, the probability of selecting A should outrank that of selecting B. If the robot then goes out of doors and onto bumpy grass, this change in the world would alter each expert's performance. As expert B outperforms expert A, the learning algorithm should respond by eventually ranking the probability of selecting B above that of selecting A.

Within Exp3, experts which have performed poorly in the past are rewarded more strongly for good performance than experts which performed well in the past. This occurs because of the ratio, within the weight update (4), which scales reward inversely with selection probability. For an expert with low selection probability (indicating poor past performance), this ratio is larger than that of an expert with high selection probability (indicating good past performance). An expert with a previously low probability of being selected, but which now performs well, may therefore have its weight increased quickly in this manner.

This adaptability idea, however, is fully captured by the Exp3 algorithm only when an expert succeeds. For example, consider instead a high probability expert which now fails, and so no longer performs as it had in the past. Its weight should represent performance under the current world conditions, and should therefore decrease. To follow the reasoning above, this decrease should occur quickly. However, in this case the scaling ratio decreases the effect of poor reward on the weight update, and the weight will actually change only minimally.

We generalize this adaptability idea by saying that expected expert performances should result in small weight changes, while unexpected expert performances should result in large weight changes. Concretely, we catagorize expert performances $s^{k}$ as successful (1) or failed (0), and selection probabilities as high $\operatorname{Pr}\left(x^{k}=i\right)>\delta_{h}$ or low $\operatorname{Pr}\left(x^{k}=i\right)<$ $\delta_{l}, 0<\delta_{l} \leq \delta_{h}<1$. We then define expert performance classifications according to Table 1 .

\begin{tabular}{|c|c|}
\hline Expected & Unexpected \\
\hline $\operatorname{Pr}\left(x^{k}=i\right)>\delta_{h} \wedge s^{k}=1$ & $\operatorname{Pr}\left(x^{k}=i\right)>\delta_{h} \wedge s^{k}=0$ \\
$\operatorname{Pr}\left(x^{k}=i\right)<\delta_{l} \wedge s^{k}=0$ & $\operatorname{Pr}\left(x^{k}=i\right)<\delta_{l} \wedge s^{k}=1$ \\
\hline
\end{tabular}

TABLE I. Classification of Expert Performance

Note the similarity between this idea and the Win or Learn Fast (WoLF) approach presented by [4] for learning agents participating in zero sum competitive games. In WoLF, agents that are loosing (ie. not performing as expected) learn at a faster rate, while those that are winning (ie. performing as expected) learn at a slower rate.

\section{B. The rExp3 Algorithm}

To further enhance adaptability, we want expert weights to update based upon whether they reflect the expected performance of their recommendation. We introduce the algorithm rExp3 (responsive Exp3) to encourage swift reactions to unexpected expert performances. In particular, our goal is to strengthen the link between selection probability and expected performance. To accomplish this, rExp3 modifies Exp3 to depend the weight updates explicitly upon expert failure or success. 
Key to our algorithm modification is to introduce two distinct formulations for the weight update multiplier, one each for whether an expert succeeds or fails. This distinction occurs in two places within the weight update; the exponent on the multiplier $m_{i}^{k}$ and the reward earned by the expert performance.

The weight update of a chosen expert $i$ at trial $k$ is determined according to the following rule,

$$
\begin{gathered}
w_{i}^{k}=w_{i}^{k-1}\left(m_{i}^{k}\right)^{\frac{1}{g}} \\
g= \begin{cases}\operatorname{Pr}\left(x^{k}=i\right) & \text { if success } \\
1-\operatorname{Pr}\left(x^{k}=i\right) & \text { if failure }\end{cases}
\end{gathered}
$$

Furthermore, we incorporate two distinct reward metrics, allowing their specific formulation to be task-determined, but requiring that a failure case reward be strictly less than 0 , and a success case reward be strictly greater than 0 . In this manner a failed execution always reduces the weight of a given expert, while a successful execution always increases the weight of a given expert. Recalling that $m_{i}^{k} \equiv e^{r_{i}^{k}}$, the strength of the exponent on this multiplier therefore determines the strength of the reward's ability to reduce or increase expert weight.

Failure-case expert outcomes are now properly represented within the weight update, such that unexpected performances have larger changes in weight than expected ones. Returning to our example of the unexpected failure of an expert with high probability, within its weight update the multiplier exponent $\left(\frac{1}{g}=\left[1-\operatorname{Pr}\left(x^{k}=i\right)\right]^{-1}\right)$ will be large. Since $0<m_{i}^{k}<1$ (due to negative reward), a larger exponent on this multiplier means a larger reduction in weight. Thus, the unexepected performance results in a significant weight reduction, as we intended.

In contrast to other learning frameworks, our direct objective is not to learn the best action for a given state. Discrete segmentation of real robot worlds can quickly produce a computationally inhibatory number of states. Instead, we learn the best expert to achieve a goal and expect that what is 'best' will change with state, without reasoning explicitly about our current state. We respond to discrete changes in state by responding to changes in expert performance. Doing so quickly is the motivation for extending Exp3 to rExp3.

\section{ALGORITHM VALIDATION ON REAL ROBOTS}

We now present results from the integration of our approach into the control architecture of a robot doing a task drawn from robot soccer. To clarify, we refer to a single execution of an expert as a trial, and to a series of trials over which learning is performed as a run.

\section{A. Robot Implementation}

The robot learning data was collected on a Segway RMP [10]. The Segway RMP is a dynamically balancing robot which in this case was outfitted to play soccer [7]. Included in our augmentations are two cameras for sensing (Fig. 3), which identify the ball for our example skill.
To illustrate the effectiveness of $r E x p 3$ on a robotic system, we applied the algorithm to the example task of learning the best ball searching routine. Seven experts were implemented, which were distinguished by the cameras from which they drew perception information to identify the ball.

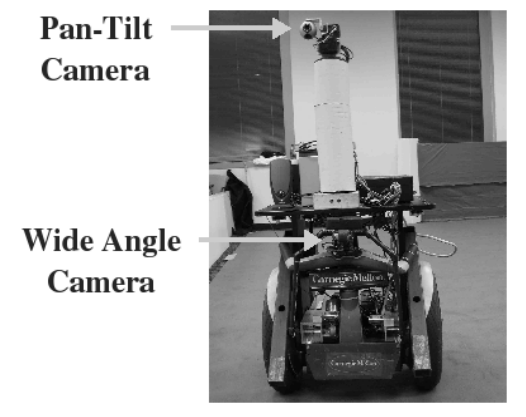

Fig. 3. Our Segway RMP soccer playing robot

Data were collected under two world conditions. In the soccer scenario the ball was passed around the robot by human riding a Segway HT, to relate learning back to the robot soccer domain. The ball therefore appeared at varying distances and states of occlusion, possibly stopped or in motion. In the occlusion scenario a controlled switch in camera occlusion was performed, to examine appropriate and quick adaptability to an environment change. By physically blocking the camera lens, the ball was visible to exclusively one camera for the first portion of a learning run, and exclusively the other for the remainder.

The reward metric for our implementation was dependent upon both expert success and execution speed. Within the context of our example skill, this represents the importance of not only finding the ball, but finding it quickly. The initial determination of success or failure was binary. The success case reward was then further subject to discounting by $\lambda$ for the $t$ time steps of execution,

$$
m_{i}^{k}= \begin{cases}1+\lambda^{t} d_{s} & \text { if success } \\ d_{f} & \text { if failure. }\end{cases}
$$

To guarantee that expert weights strictly increase with successful executions, and strictly decrease with failed executions, we require $d_{s}>0$ and $0<d_{f}<1$ (here $\lambda=$ $\left.0.98, d_{s}=0.3, d_{f}=0.7\right)$. For implementation simplicity, we dealt directly with the multiplier $m_{i}^{k} \equiv e^{r_{i}^{k}}$ present in our weight update, rather than the actual reward $r_{i}^{k}$.

\section{B. Robot Results}

The algorithm rExp3 was able to properly and flexibly learn which ball search routines to execute.

Within the soccer scenario, the experts preferred by the learning algorithm agreed with those which performed fastest, as determined by baseline data collected without learning. From this baseline data we qualitatively classify the experts into three performance catagories, where Experts 0-2 perform well, Experts 3-4 perform moderately, and Experts 5-6 perform poorly. Note that these classifications apply only to soccer scenario world conditions, and will not necessarily hold when cameras are obstructed within 
the occlusion scenario. Figure 4A presents a single example learning run, where the learned expert (Expert 1) was also one which performed well during baseline data collection.

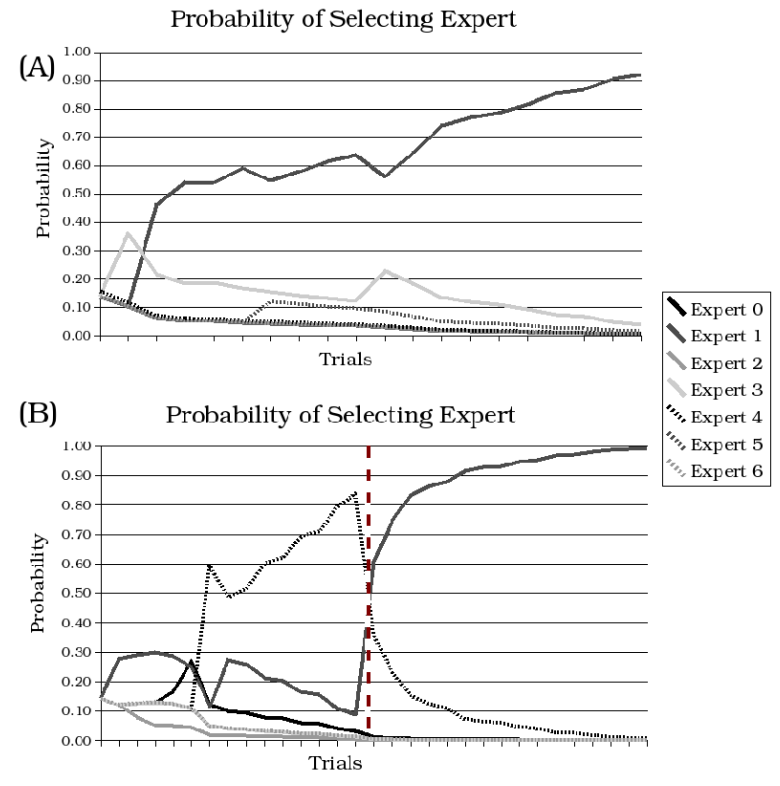

Fig. 4. Single rExp3 robot learning runs, soccer (A) and occlusion (B) scenarios. The vertical line in $\mathrm{B}$ indicates a discrete environment change.

In the occlusion scenario, the algorithm $r E x p 3$ was able to recover from the switch in occluded camera $(1.75 \pm 0.5$ trials to recover). Figure $4 \mathrm{~B}$ presents a single example learning run, where at first Expert 5 dominates. Expert 5 is an expert which depends upon the camera which will be occluded after the switch (vertical line). Following the switch, accordingly, an expert which does not depend upon this camera (Expert 1) comes to dominate.

\section{ALGORITHM VALIDATION IN SIMULATION}

To further test the performance of $r \operatorname{Exp} 3$, both of the algorithms $r$ Exp3 and Exp3 were implemented in simulation. Our goal was that the modification introduced in rExp3 would compare to Exp3 in the following manner:

- Faster recovery (defined below), when the performance was unexpected.

- Lower regret, when the performance was unexpected.

- Similar regret, when the performance was expected.

\section{A. Simulation Implementation}

Within our simulation implementation, experts recommended actions in worlds constructed such that their performance might clearly be classified as expected or unexpected.

Specifically, our simulation mimicked the ball searching task of the real robot implementation. There were again seven experts distinguished by the camera combinations from which they drew perception information. Each expert had an associated failure probability $\operatorname{Pr}\left(x^{k}=0\right)$, which was determined by the world and based upon whether a currently occluded camera was depended upon by the expert. All learning parameters were set as in the real robot implementation.
Our intent was to gather data at a simulated environment switch, but to do so in a controlled manner. We therefore began our data collection at this switch, and simulated prior learning by biasing the initial selection probabilities to favor a single expert. Each expert consequently had an associated initial selection probability $\operatorname{Pr}\left(x^{0}=i\right)$ as well as its failure probability, the combination of which determined the classification of expert performance as expected or unexpected according to Table 1 .

\section{B. Simulation Results}

In the comparison of $r \operatorname{Exp} 3$ to Exp3, our simulation results show both faster recovery and lower regret for unexpected performances, as well as similar regret for expected performances. Data were collected over 100 runs of 50 trials each for expected and unexpected scenarios, as well as 50 runs of 50 trials with no learning, to provide a baseline against which each learning algorithm might be compared.

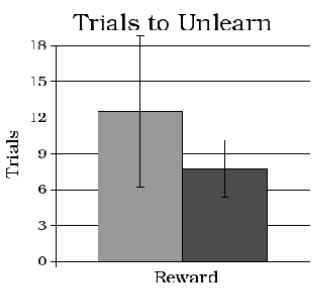

(A)

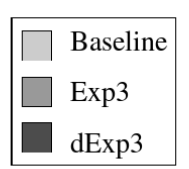

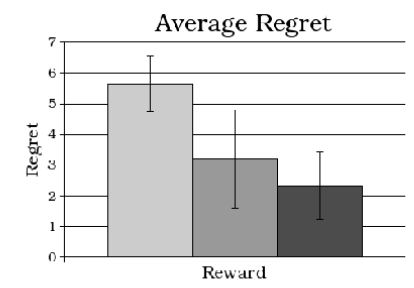

(B)

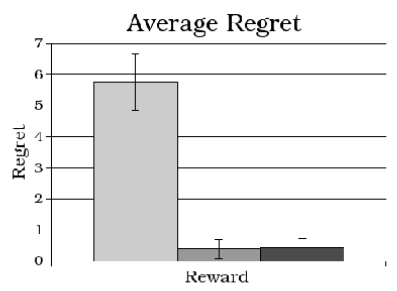

(C)
Fig. 5. Average results of baseline (no learning), Exp3 and rExp3 simulation learning data. Unexpected performance, trials to recovery (A) and average regret (B); expected performance average regret (C). Error bars for all show one standard deviation.

1) Faster Recovery (Unexpected Performances): Significantly fewer time steps were required for $r E x p 3$, compared to Exp3, to respond to a previously well performing expert which now fails (trials to recover for $r E x p 3=7.74$ vs. Exp3 $=12.52, t=4.14,99.9 \%$ confidence interval $>3.26$, Fig. 5A). Recovery was defined as the time step at which a favored expert no longer had the highest probability of being selected.

2) Lower Regret (Unexpected Performances): The overall regret of $r$ Exp3 was significantly lower than that of Exp3 when an expert performed unexpectedly, particularly when an expert which previously performed well began to fail (regret on $r \operatorname{Exp} 3=2.33$ vs. Exp3 $=3.21, t=3.1,99.5 \%$ confidence interval $2.68-3.26$, Fig. 5B). Both learning algorithms perform significantly better than the baseline data which utilized no learning $(r E x p 3 t=18.39$, Exp3 $t=9.24,99.9 \%$ confidence interval $>3.26)$. 
3) Similar Regret (Expected Performance): The difference in regret between the two algorithms was not significant when experts exhibited expected performances and required no relearning (regret on $r \operatorname{Exp} 3=0.44$ vs. Exp3 $=0.39$, $t=0.81,75.0 \%$ confidence interval $0.68-1.30$, Fig. 5C). By contrast, the difference in regret between each learning algorithm and the baseline data which used no learning was significant for each $(r E x p 3 t=39.05, \operatorname{Exp} 3 t=$ $38.82,99.9 \%$ confidence interval $>3.26)$.

\section{DISCUSSION}

The algorithm rExp3 modifies Exp3 by addressing expert failure or success explicitly within the weight update. In adopting two distinct reward scalings and exponent formulations, our intent is to strengthen the link between expert performance and selection probability.

Relating this modification back to the original inverse scaling motivation of Auer and colleagues, the failure case scaling for a high probability expert might be seen as a prediction on future reward frequency. A high probability expert which begins to fail also begins to drop in selection probability. Scaling with $\frac{1}{1-\operatorname{Pr}\left(x^{k}=i\right)}>\frac{1}{\operatorname{Pr}\left(x^{k}=i\right)}$ in a sense already begins to compensate for the predicted fewer future selections of this expert, and therefore also for the decrease in future reward observations. The same may be reasoned about the inverse scaling with $\operatorname{Pr}\left(x^{k}=i\right)$ in the low probability success case, present within both algorithms.

Past work with Exp3 tested the algorithm in environments different to those presented here, namely against an allknowing adversary [1]. If tested in similar environments to the past work of Exp3, we expect dExp3 would perform at least as well as Exp3. Our reasoning is twofold. First, in our simulation results for scenarios with no explicit unexpected performances, the algorithms responded similarly. Second, particularly within the context of an all knowing adversary, dExp3 would adapt quicker to those changes in the adversary's strategies which resulted in the poor performance of a previously successful expert. Worst case scenario would be if the adversary altered strategy at a rate such that the change in relative expert weights was strong within $r E x p 3$, but relatively stable within Exp3. Under such circumstances, however, we expect that the strategy of the adversay would be changing too quickly for any learning to prove useful, in which case we again would expect similar performances.

It is possible during execution that an expert might oscillate between failure and success. Within the weight update of the rExp3 formulation, this will then induce an oscillation in exponent formulation. One argument might favor such an oscillation, as it represents the true behavior of the expert. Indeed, as our intent for this algorithm is very domain specific, we value swift responses to our dynamic soccer environment. By contrast, other approaches might attempt to minimize responses to domain instability. Note, however, that an oscillation in exponent formulation will not necessarily cause an oscillation in the actual weight. In fact, such a weight oscillation will only occur if the selection probability of an expert lies at either extreme. From our empirical observations, such extreme oscillations rarely occured.

\section{CONCLUSIONS}

We have presented an adapted experts approach for learning the execution control loop on a robot system, and demonstrate its effectiveness on a real robot system. We introduce a modified experts learning algorithm, which we call rExp3, based upon the Exp3 algorithm of Auer and colleagues [1], to enhance responsiveness to discrete environment changes. The effectiveness of this modification is presented with comparisons between rExp3 and Exp3 within simulation, in addition to the implementation of rExp3 on a Segway robot. When a learned good expert begins to fail, we have shown rExp3 to both accumulate smaller overall regret and 'unlearn' this expert faster, and to behave similarly to Exp3 otherwise. In future work, we intend to apply this algorithm to skill selection in other soccer scenarios, particularly when in response to strategy decisions of the opponent team.

\section{ACKNOWLEDGMENTS}

This work was supported by United States Department of the Interior under Grant No. NBCH-1040007. The content of the information in this publication does not necessarily reflect the position or policy of the Defense Advanced Research Projects Agency (DARPA), US Department of Interior, US Government, and no official endorsement should be inferred.

\section{REFERENCES}

[1] Peter Auer, Nicol'o Cesa-Bianchi, Yoav Freund, and Robert E. Schapire. Gambling in a rigged casino: The adversarial multiarm bandit problem. In 36th Annual Symposium on Foundations of Computer Science, pages 322-331, Milwaukee, WI, 1995.

[2] T. Balch, G. Boone, T. Collins, H. Forbes, D. MacKenzie, and J. Santamaria. Io, ganymede and callisto: A multiagent robot trashcollecting team. AI Magazine, 16(2):39-53, 1995.

[3] Michael Bowling, Brett Browning, and Manuela Veloso. Plays as effective multiagent plans enabling opponent-adaptive play selection. In Proceedings of International Conference on Automated Planning and Scheduling (ICAPS'04), 2004. in press.

[4] Michael Bowling and Manuela Veloso. Convergence of gradient dynamics with a variable learning rate. In Proceedings of ICML-2001, pages 27-34, Williams College, MA, June 2001.

[5] R. A. Brooks. A robust layered control system for a mobile robot. IEEE Journal on Robotics and Automation, RA-2(1), 1986.

[6] B. Browning, J. Bruce, M. Bowling, and M. Veloso. STP: Skills, tactics and plays for multi-robot control in adversarial environments. IEEE Journal of Control and Systems Engineering, 2004.

[7] B. Browning, P. Rybski, J. Searock, and M. Veloso. Development of a soccer-playing dynamically-balancing mobile robot. In Proceedings of International Conference on Robotics and Automation, May 2004.

[8] Yoav Freund and Robert E. Schapire. A decision-theoretic generalization of on-line learning and an application to boosting. Journal of Computer and System Sciences, 55:119-139, 1997.

[9] Nick Littlestone and Manfred K. Warmuth. The weighted majority algorithm. Information and Computation, 108:212-261, 1994.

[10] H. G. Nguyen, J. Morrell, K. Mullens, A. Burmeister, S. Miles, K. Thomas, and D. W. Gage. Segway robotic mobility platform. In SPIE Mobile Robots XVII, October 2004.

[11] Herbert Robbins. Some aspects of the sequential design of experiments. Bulletin American Mathematical Society, 55:527-535, 1952.

[12] Reid Simmons and David Apfelbaum. A task description language for robot control. In Proceedings of Conference on Intelligent Robotics and Systems, Vancouver Canada, October 1998.

[13] Volodimir G. Vovk. Aggregating strategies. In Proceedings of the Third Annual Workshop on Computational Learning Theory, pages 371-383, 1990. 